\title{
Histone Deacetylase Inhibition via RGFP966 Releases the Brakes on Sensory Cortical Plasticity and the Specificity of Memory Formation
}

\author{
Kasia M. Bieszczad, ${ }^{1,2,3,4}$-Kiro Bechay, ${ }^{1}$ James R. Rusche, ${ }^{5}$ Vincent Jacques, ${ }^{5}$ Shashi Kudugunti, ${ }^{5}$ Wenyan Miao, ${ }^{5}$ \\ Norman M. Weinberger, ${ }^{1,2}$ James L. McGaugh, ${ }^{1}$ and Marcelo A. Wood ${ }^{1,2}$ \\ ${ }^{1}$ Department of Neurobiology and Behavior, Center for the Neurobiology of Learning and Memory and ${ }^{2}$ Center for Hearing Research, University of \\ California, Irvine, California 92697, ${ }^{3}$ College for Life Sciences, Wissenschaftskolleg zu Berlin, 14193 Berlin, Germany, ${ }^{4}$ Psychology Department, Behavioral \\ and Systems Neuroscience, Rutgers, The State University of New Jersey, Piscataway, New Jersey 08854, and ${ }^{5}$ Repligen Corporation, Waltham,
}

Massachusetts 02453

Research over the past decade indicates a novel role for epigenetic mechanisms in memory formation. Of particular interest is chromatin modification by histone deacetylases (HDACs), which, in general, negatively regulate transcription. HDAC deletion or inhibition facilitates transcription during memory consolidation and enhances long-lasting forms of synaptic plasticity and long-term memory. A key open question remains: How does blocking HDAC activity lead to memory enhancements? To address this question, we tested whether a normal function of HDACs is to gate information processing during memory formation. We used a class I HDAC inhibitor, RGFP966 $\left(\mathrm{C}_{21} \mathrm{H}_{19} \mathrm{FN}_{4} \mathrm{O}\right)$, to test the role of HDAC inhibition for information processing in an auditory memory model of learning-induced cortical plasticity. HDAC inhibition may act beyond memory enhancement per se to instead regulate information in ways that lead to encoding more vivid sensory details into memory. Indeed, we found that RGFP966 controls memory induction for acoustic details of sound-toreward learning. Rats treated with RGFP966 while learning to associate sound with reward had stronger memory and additional information encoded into memory for highly specific features of sounds associated with reward. Moreover, behavioral effects occurred with unusually specific plasticity in primary auditory cortex (A1). Class I HDAC inhibition appears to engage A1 plasticity that enables additional acoustic features to become encoded in memory. Thus, epigenetic mechanisms act to regulate sensory cortical plasticity, which offers an information processing mechanism for gating what and how much is encoded to produce exceptionally persistent and vivid memories.

Key words: auditory cortex; chromatin modification; cortical plasticity; epigenetics; histone acetylation; memory

\section{Significance Statement}

Here we provide evidence of an epigenetic mechanism for information processing. The study reveals that a class I HDAC inhibitor (Malvaez et al., 2013; Rumbaugh et al., 2015; RGFP966, chemical formula $\mathrm{C}_{21} \mathrm{H}_{19} \mathrm{FN}_{4} \mathrm{O}$ ) alters the formation of auditory memory by enabling more acoustic information to become encoded into memory. Moreover, RGFP966 appears to affect cortical plasticity: the primary auditory cortex reorganized in a manner that was unusually "tuned-in" to the specific sound cues and acoustic features that were related to reward and subsequently remembered. We propose that HDACs control "informational capture" at a systems level for what and how much information is encoded by gating sensory cortical plasticity that underlies the sensory richness of newly formed memories.

\section{Introduction}

Histone modification is dynamically regulated during memory formation (Peixoto and Abel, 2013), yet the impact of such epi-

Received March 9, 2015; revised Aug. 15, 2015; accepted Aug. 18, 2015.

Author contributions: K.M.B., N.M.W., J.L.M., and M.A.W. designed research; K.M.B. performed research; K.M.B., J.R.R., V.J., and S.K. contributed unpublished reagents/analytic tools; K.M.B., K.B., S.K., and W.M. analyzed data; K.M.B. and M.A.W. wrote the paper. genetic mechanisms on information encoding has scarcely been addressed. Stefanko et al. (2009) introduced the idea that histone

This work was supported by grants from the NIDCD (N.M.W., K.M.B.), NIMH (J.L.M., M.A.W.), and NIDA (M.A.W.) and the Wissenschaftskolleg zu Berlin Institute for Advanced Study (Berlin, Germany) (K.M.B.). V.J., S.K. and W.M. are employees of Repligen Corporation. We thank Erica Gregor and Drs. Teiko Miyashita, Melissa Malvaez, and Annie Vogel-Ciernia for technical advice and assistance. We also acknowledge our colleagues in the related fields of epigenetics, learning and memory, and of auditory cortical function whose papers we could not cite due to space limitations. 
modification induced by histone deacetylase (HDAC) inhibitors can transform a learning event that would not normally lead to memory into one that does. Moreover, these memories are in a form that persists beyond the point at which natural memory fails. Effects of HDAC inhibitors thus appear to act beyond memory enhancement, which would be expected to simply "boost" the strength of memory that would have naturally formed. Instead, these findings give rise to the following question: Do HDACinhibitors produce a dramatic change in information processing itself to alter what and how much information becomes encoded to produce persistent and vivid memory? If so, a much more significant role for HDAC enzymes exists in learning and memory than previously realized.

Here, we focused on the class I histone deacetylase inhibitor called RGFP966 $\left(\mathrm{C}_{21} \mathrm{H}_{19} \mathrm{FN}_{4} \mathrm{O}\right)$. Based on previously published recombinant purified protein assays in vitro, RGFP966 may have selectivity for HDAC3 over HDAC1 and HDAC2 (Malvaez et al., 2013). Class I HDACs include HDAC1, HDAC2, HDAC3, and HDAC8. HDAC3 is the most highly expressed class I HDAC in the brain and a key negative regulator of gene expression required for long-term memory formation (McQuown and Wood 2011; McQuown et al., 2011). Previous evidence suggests a role for HDAC3 in consolidation effects, since application of the selective HDAC3 inhibitor used here (RGFP966) does not itself induce synaptogenesis, nor can it rescue memory impairment in an animal model of Alzheimer's disease (Rumbaugh et al., 2015). Rather, HDAC3 appears to require a learning event to act on memory and promote synaptic plasticity (Rumbaugh et al., 2015). Indeed, the HDAC3 inhibitor RGFP966 has been shown to act on "subthreshold" experiences that normally would not have been remembered, transforming them into incredibly robust and persistent long-term memories (McQuown et al., 2011; Malvaez et al., 2013). Although several studies have examined the role of HDAC3 and other HDACs in memory processes, none have examined the role of HDACs in regulating information processing in the auditory cortex $(\mathrm{AC})$, which allows for unique approaches to understand how HDACs regulate information being encoded into long-term memory.

This is the first study to address the function of class I HDACs in information encoding for auditory memory and learninginduced cortical plasticity. The primary auditory cortex (A1) is now appreciated for its functions in learning and memory. Plasticity in Al has become the most extensively studied of sensory areas for its function in associative memory (Weinberger, 2007; Scheich and Ohl, 2010; Pienkowski and Eggermont, 2011; Schreiner and Polley, 2014). For example, learning-induced tonotopic map expansion appears to enable both the strength of auditory memory formation and the specificity for what sounds and sound features are encoded into memory (Bakin and Weinberger 1990; Schulte et al., 2002; Bao et al., 2004; Rutkowski and Weinberger, 2005; Polley et al., 2006; Bieszczad and Weinberger 2010a, 2012; Lee and Middlebrooks, 2011), including the instantiation of sound-specific memory when tonotopic plasticity is artificially induced (Bieszczad et al., 2013; Froemke et al., 2013;

The authors declare no competing financial interests.

Correspondence should be addressed to either of the following: Dr. Kasia M. Bieszzzad, Department of Psychology, Psychology Building, Room 227, Busch Campus, 152 Frelinghuysen Road, Rutgers University, Piscataway, NJ 08854, E-mail: kasia.bie@rutgers.edu; or Dr. Marcelo A.Wood, Department of Neurobiology and Behavior, Center for the Neurobiology of Learning and Memory, 301 Qureshey Laboratory, University of California, Irvine, CA 92697, E-mail:mwood@uci.edu.

DOI:10.1523/JNEUROSCI.0914-15.2015

Copyright $\odot 2015$ the authors $\quad$ 0270-6474/15/3513125-09\$15.00/0
Weinberger et al., 2013). Indeed, A1 plasticity might enable specific auditory features of a learning experience to become encoded for a persistent and vivid memory. We applied an auditory model to understand how an HDAC3-selective inhibitor could change information processing for what and how much becomes encoded into memory by engaging A1 plasticity.

\section{Materials and Methods}

Animals

A total of thirty-three adult male Sprague Dawley rats (275-350 g) were used in behavioral, electrophysiological, and/or molecular experiments. All animals were housed in an Association for Assessment and Accreditation of Laboratory Animal Care International accredited, temperaturecontrolled $\left(22^{\circ} \mathrm{C}\right)$ facility on a $12 \mathrm{~h}$ light/dark cycle. Procedures were performed according to guidelines approved by the University of California Irvine's Institutional Animal Care and Use Committee and in accordance with the National Institutes of Health Office of Laboratory Animal Welfare guidelines. An illustration of the experimental timeline and training procedure is provided in Figure 1.

\section{Behavioral training}

Water-restricted rats were trained to associate sounds with water reward (Fig. 1B, C). The protocol involved a task [developed by Bieszczad and Weinberger (2010a)] that required animals to press a bar manipulandum upon hearing a pure tone (Fig. $1 B$ ). Two sounds could be associated with reward. The first, Signal A (5.0 kHz, $70 \mathrm{~dB}$ SPL), was a pure tone sound that signaled the availability of reward upon the subject performing a bar-press (BP) response within $3 \mathrm{~s}$ of Signal A onset. The second, Signal $\mathrm{B}$, was the sound of the reward delivery lever mechanism (with significant power in the 0.1 to $2.0 \mathrm{kHz}$ sound frequency band; see Rutkowski and Weinberger, 2005). This signaled the reward's immediate delivery. Acquisition was determined by performance level, calculated for each session throughout training as the percentage of bar presses within tone presentations that occurred only within the first $3 \mathrm{~s}$ of tone onset (rewarded BPs), scaled by the percentage of bar presses within a session that occurred at any time during tone presentations: performance $=[$ (number rewarded BPs/number tone BPs) $\times$ (number tone BPs/number total $\mathrm{BPs}) \times 100 \%]$. All animals trained successfully learned to associate sound with reward (Fig. 1C).

\section{Behavioral memory test}

A memory test after training (Fig. $1 B$ ) revealed the frequency specificity of the auditory memory by determining behavioral responses to many frequencies, including that of Signal A $(5.0 \mathrm{kHz})$ and Signal B $(1.1 \mathrm{kHz})$. Bar-press response latencies to the onset of each test frequency $(1.1,2.4$, $5.0,10.6$, and $22.4 \mathrm{kHz}$, each presented at $70 \mathrm{~dB}$ SPL) were determined in a single session of 200 unrewarded trials ( 40 trials of each test frequency, pseudorandomly intermixed). Response latency for each test tone was determined as the average latency from tone onset until a bar press for trials in which at least one bar press occurred. This latency was normalized within animal by its own average latency to press across all session trials with at least one bar press. These normalized values were used to construct a behavioral gradient to index memory specificity in the dimension of acoustic frequency for each animal, and then for the group. A distinct shorter latency response (see below, Determining behavioral frequency specificity, Individual analysis) was used as a proxy for greater frequency specificity.

\section{Drug administration}

The critical manipulation was pharmacological inhibition using a class I HDAC inhibitor called RGFP966. Immediately following the daily training session, a posttraining systemic injection of either RGPF966 (10 mg/ $\mathrm{kg}$, s.c.) or vehicle (at a comparable volume to drug treatment) was delivered to each subject. The effective $10 \mathrm{mg} / \mathrm{kg}$ dose of RGFP966 (the uniquely selective HDAC3 inhibitor) was established previously in mice (Malvaez et al., 2013) and confirmed to penetrate the blood-brain barrier to rat auditory cortex with similar pharmacokinetics (Fig. $1 A$ ), which here establishes the rationale for a single-dose, single-inhibitor approach. Furthermore, the effects of postsession doses of RGFP966 for HDAC 
A

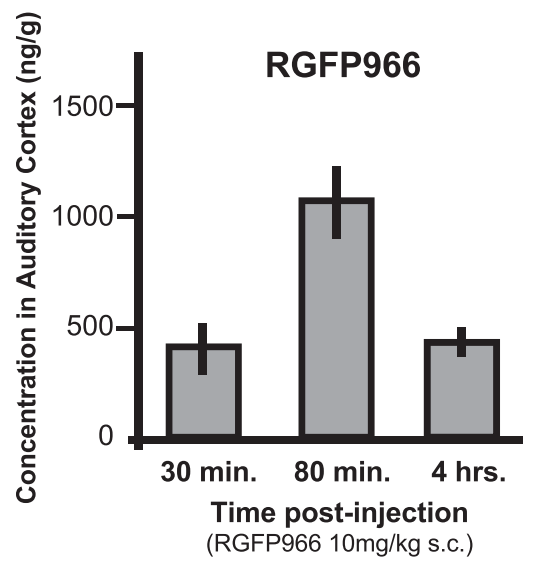

C

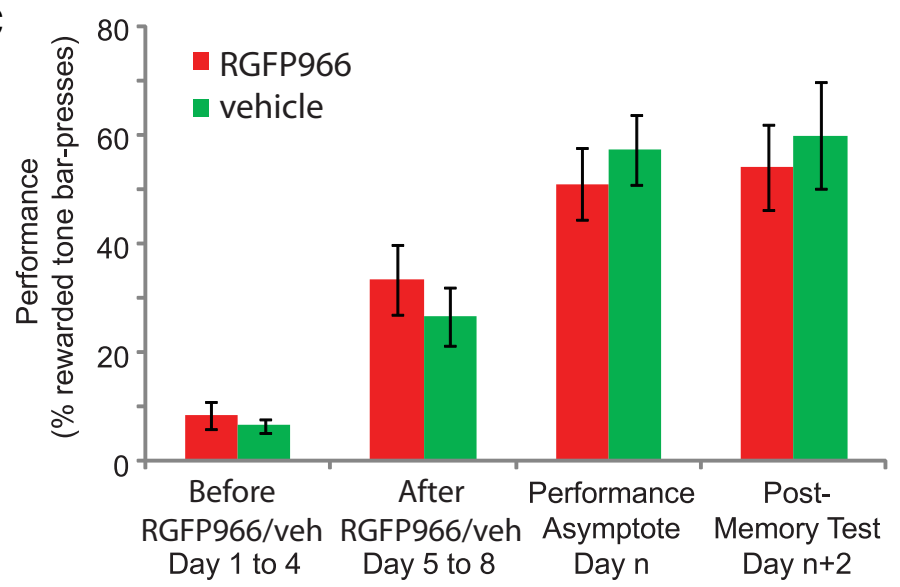

B

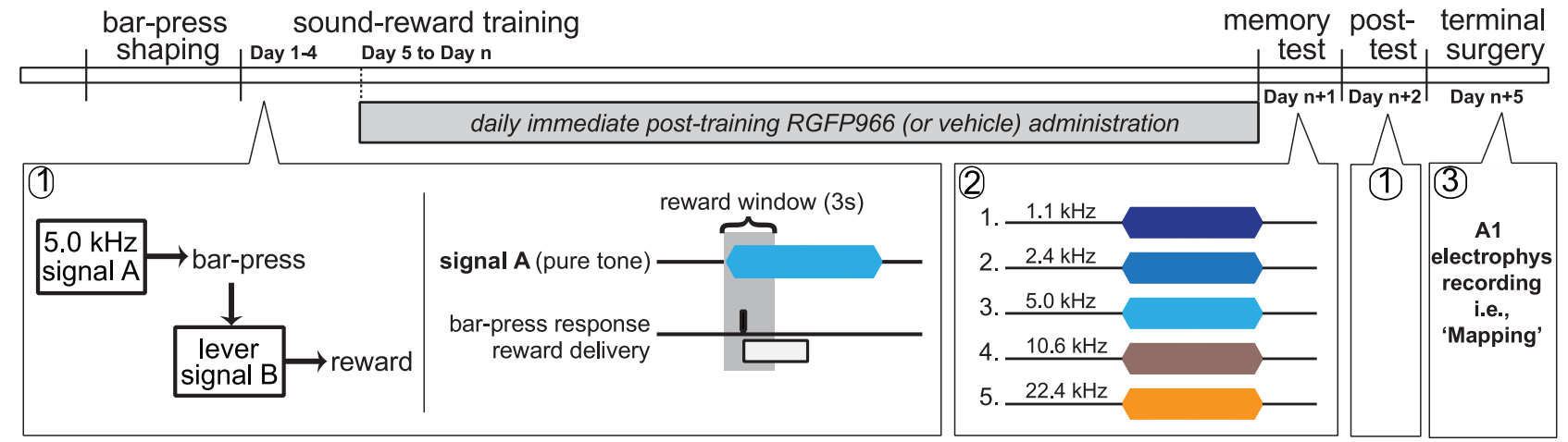

Figure 1. Protocol and timeline for auditory learning, memory testing and determining A1 plasticity. A, RGFP966 kinetics in auditory cortex. The effective $10 \mathrm{mg} / \mathrm{kg}$ dose of RGFP966 (a selective HDAC3 inhibitor) was established by Malvaez et al. (2013) and confirmed here to penetrate the blood-brain barrier $\left(C_{\max }=415 \mathrm{ng} / \mathrm{g} \pm 120\right.$ at $30 \mathrm{~min} ; C_{\max }=1065 \mathrm{ng} / \mathrm{g} \pm 163$ at 75 min; $C_{\max }=451 \mathrm{ng} / \mathrm{g} \pm 54$ at $4 \mathrm{~h}$ ). Its action in rat auditory cortex occurs with similar pharmacokinetics to treatments with RGFP966 that are known to modulate memory formation and persistence (Malvaez et al., 2013). Data shown are mean \pm SEM ( $N=3$ untrained animals) for each time point. Inset, Chemical structure of RGFP966. B, All animals were trained to associate sound with reward in an instrumental conditioning paradigm (sound-reward training). Signal A was a pure tone ( $5.0 \mathrm{kHz}, 70 \mathrm{~dB} \mathrm{SPL}$ ) that predicted the availability of reward after a bar-press response. Signal B was a sound typical of behavioral training equipment that was produced by a mechanical lever holding reward; it predicted the immediate delivery of reward on correct trials (1-2 kHz). After training, the frequency specificity of associative memory was determined using various test tones (including Signal A, $5.0 \mathrm{kHz}$, and Signal B, $1.1 \mathrm{kHz}$ ) to indicate which frequency elicited reward-related bar-pressing behavior (memory test). Rewards were omitted during this session. Frequency tuning and tonotopic representation in A1 were determined by electrophysiological recording (A1 mapping). C, Performance on the auditory association task was not affected by RGFP966. There are no significant differences between groups before treatment with RGFP966 (days $1-4$ ) and after treatment (days $5-8$; before, RGFP966, $8.5 \pm 2.5 \%$ vs vehicle, $6.4 \pm 1.4 \% ; p=0.248$; after, RGFP966, $33.3 \pm 6.4 \%$ vs vehicle, $26.6 \pm 5.2 \% ; p=0.101 ; N=6$ for each group). Likewise, asymptotic levels of performance immediately preceding the memory test are not significantly different between groups (RGFP966, $N=6,51.1 \pm 6.5 \%$ vs vehicle, $N=6,57.4 \pm 6.5 \% ; p=0.284$ ). RGFP966 similarly did not affect the memory test session, which was without any rewards, as determined by a reinstatement session (i.e., a session identical to training and with rewards) the day

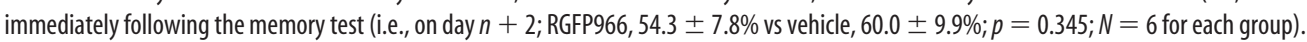

inhibition are expected to act only on associative mechanisms of memory consolidation. Administration of RGFP966 (or vehicle) began after $4 \mathrm{~d}$ of initial sound-reward training to ensure all animals had initially acquired an auditory association with reward. This established an initial acquisition curve, which was also useful to determine performance-matched pairs of RGFP966- and vehicle-treated rats used in subsequent analysis of neural plasticity.

Distribution of RGFP966 in auditory cortex

Additional untrained animals $(N=9)$ were dosed with RGFP966 (10 $\mathrm{mg} / \mathrm{kg}$, s.c.) and killed at 30,80 , and $240 \mathrm{~min}$ after administration $(N=3$ per time point). Brains were harvested and flash frozen. Samples from the auditory cortex were blotted and weighed. Water containing $0.1 \%$ formic acid at a ratio of 5:1 (v/w) was added into the auditory cortex tube. For example, $1000 \mu \mathrm{l}$ of $0.1 \%$ formic acid was added to a $200 \mathrm{mg}$ tissue. After tissue homogenization, samples were mixed with acetonitrile containing an internal standard to precipitate protein and were then centrifuged. Concentrations of RGFP966 in supernatants were determined by liquid chromatography mass spectrometry [API-4000 triple quadrupole (AB Sciex) equipped with a CTC Pal autosampler (Leap Technologies) and an Agilent 1100 high-performance liquid chromatograph]. Samples were analyzed using the multiple reaction monitoring quantitation method, in which signals resulting from a specific parent-daughter ion transition, specifically 364.2-236.8 atomic mass unit for RGFP966, were measured after separation of the sample on an HPLC column $(50 \times 4.6 \mathrm{~mm}, 5 \mu \mathrm{m}$ Gemini C18 column; Phenomenex) using a gradient of $0.1 \%$ formic acid in water and $0.1 \%$ formic acid in acetonitrile from 95:5 to 5:95 (v/v). Peak areas of RGFP966, normalized to an internal standard, were quantified from the regression equation (quadratic regression with $1 / x^{2}$ weighting) obtained by quantification of standards prepared from stock solution of drug diluted in blank rat brain (Bioreclamation) and treated as described above for unknown samples. All analyses and calculations were performed using Analyst software (AB Sciex; Fig. 1A).

\section{Electrophysiological recording}

The tonotopy and borders of A1 were determined electrophysiologically in an acute "mapping" session performed at the termination of all training and testing from an anesthetized animal (sodium pentobarbital, 50 $\mathrm{mg} / \mathrm{kg}$, i.p.) that has been a standard preparation in cortical neurophysiological research (Fig. 1B). Combinations of frequency and intensity sounds $(0.75-54.0 \mathrm{kHz}$ in quarter-octave steps; $0-70 \mathrm{~dB}$ SPL in $10 \mathrm{~dB}$ steps; six pseudorandom repetitions) from a speaker at the entrance to the contralateral ear canal were used to evoke responses recorded from an array $(1 \times 4)$ of Parylene-coated tungsten electrodes to layers IV-V (400-600 $\mu \mathrm{m}$ depth; FHC; $1-2 \mathrm{M} \Omega$ ). Electrodes were placed at locations that covered the surface of the auditory cortex, $\sim 250 \mu \mathrm{m}$ apart at each 
A
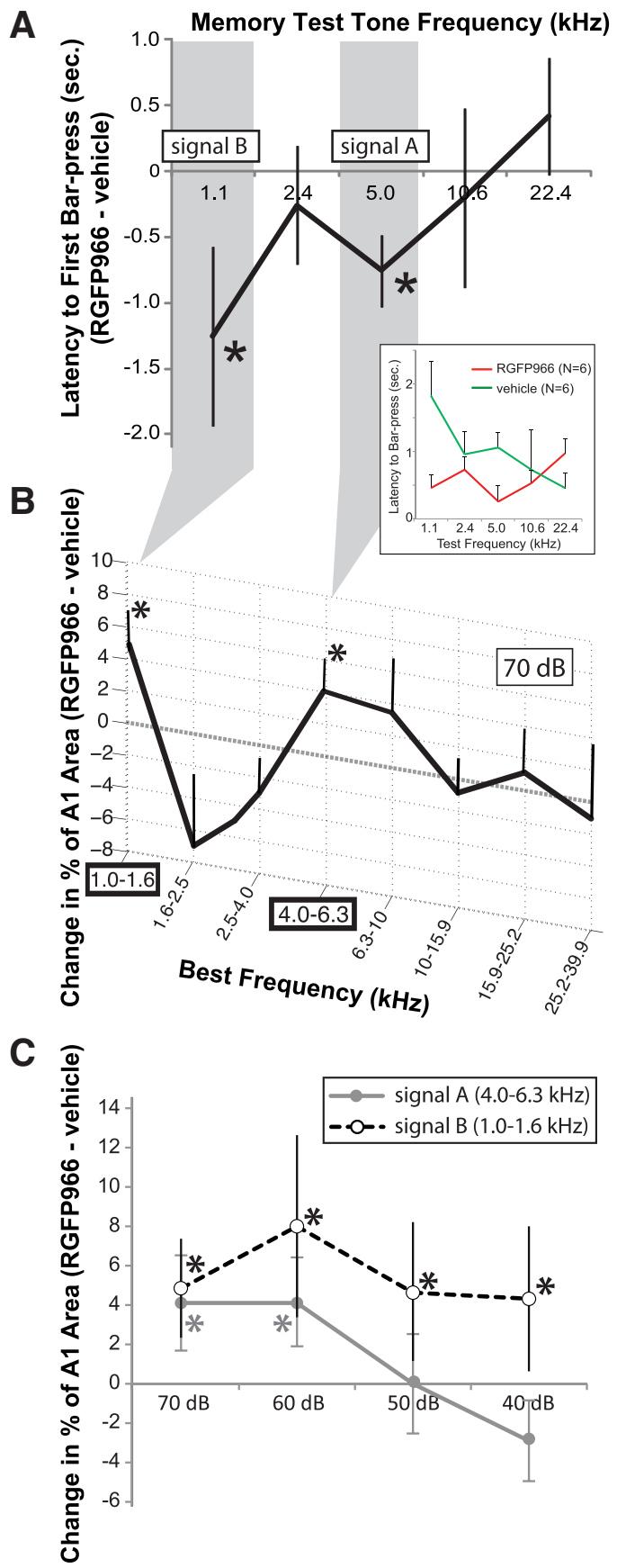

Figure 2. RGFP966 enables highly specific memory and A1 plasticity. $A$, Behavior. The latency to bar press for each test tone frequency was determined in each group to indicate which frequencies best elicited reward-related behavior. Rats treated with RGFP966 $(N=6)$ were faster to respond than performance-matched rats treated with vehicle alone $(N=6)$ to frequencies associated with reward (Signal A) and reward delivery [Signal B; Z-score for paired difference in latency to respond to each test frequency; calculated as RGFP966 minus vehicle, responses to $1.1 \mathrm{kHz}$ (Signal B) were on average $1.25 \pm 0.7 \mathrm{~s}$ significantly faster, $p=0.030$; responses to $2.4 \mathrm{kHz}$ were $0.26 \pm 0.5 \mathrm{~s}$ faster, $p=0.280$; responses to $5.0 \mathrm{kHz}$ (Signal A) were $0.75 \pm 0.3$ s significantly faster, $p=0.003$; responses to $10.6 \mathrm{kHz}$ were $0.75 \pm 0.3 \mathrm{~s}$ faster, $p=$ 0.380 ; responses to $22.4 \mathrm{kHz}$ were $0.42 \pm 0.5 \mathrm{~s}$ slower, $p=0.830$ ), which indicates the formation of a more highly specific memory for acoustic frequency with RGFP966. Inset, Mean ( \pm SE) bar-press latencies show group response gradients for the RGFP966 $(N=6)$ and vehicle $(N=6)$ groups, without respect to performance-matched pairs. $B$, Sound frequency representation in A1. Rats treated with RGFP966 had greater expansions of best frequency areas in A1 to overrepresent sound frequencies near the reward, Signal A ( $5.0 \mathrm{kHz}$; in the $4.0-6.3 \mathrm{kHz}$ frequency band) and reward delivery signal, Signal B (1.1 kHz; in the 1.0 -1.6 kHz frequency band). Asterisks indicate frequency bands that were significant with both paired (sign test) and unpaired (Mann-Whitney-Wilcoxon) one-sided tests after Holm-Bonferroni correction cortical site, to identify responses in A1 ( $~ 68-92$ recording sites in total per animal). Short-latency evoked responses within 6-40 ms of stimulus onset (typical of primary cortical responses) were used to identify a characteristic frequency (CF; frequency to which the site is most sensitively tuned, i.e., at threshold) using custom algorithms processed in Matlab. A general progression of increasing CFs from posterior to more anterior locations across the cortical surface identified the anteroposterior (AP) borders of A1. This mapping approach has been critical for identifying the perimeters of $\mathrm{Al}$ and performing a comprehensive analysis of its tuning characteristics like receptive field bandwidths (BWs) and bestfrequency $(\mathrm{BF})$ tuning, including the tonotopic distribution of $\mathrm{BF}$ and CF representation (Merzenich et al., 1975; Gonzalez-Lima and Scheich, 1986; Rutkowski et al., 2003; Polley et al., 2007; Bieszczad and Weinberger, 2010a,b).

\section{Analysis of A1}

Evoked responses were averaged across stimulus repetitions to construct frequency response areas (FRAs) that reveal the CF, evoked threshold at $\mathrm{CF}$ (CF threshold), $\mathrm{BF}$ (i.e., best tuning above threshold), and tuning BW (e.g., $20 \mathrm{~dB}$ SPL above threshold) for each A1 site. The representational area in A1 was measured using a Voronoi tessellation algorithm (based upon the voronoi algorithm in Matlab), which approximates twodimensional areas in polygons labeled by CF (or BF) at each site that together delineate the cortical surface into regional areas of frequency representation. Areas were defined per isofrequency band, i.e., defined by the area contained by CFs or BFs within a half-octave range. Betweengroup $Z$-scores for differences from zero were used to contrast frequency specificity of A1 reorganization. Differences in the magnitude of A1 area and bandwidth changes were determined in performance-matched pairs of HDAC3 inhibition (HDAC3i)- versus vehicle-treated animals. The data shown in Figures 2 and 3 show the means of these differences between groups. Figure 3 uses a three-dimensional axis to combine the difference data presented separately for frequency and sound level in Figure 2 to give a comprehensive picture of the remodeled landscape of A1 sound representation. Tuning bandwidths were defined for each group per isofrequency band, i.e., defined by the bandwidth of averaged FRAs grouped by CFs within a half-octave range. Group mean bandwidth was determined with respect to threshold, at 10, 20, 30, and $40 \mathrm{~dB}$ SPL above CF threshold. Bandwidths were compared between groups at each level above threshold for each isofrequency pop-

$\leftarrow$

( $p$ values for sign test/Mann-Whitney-Wilcoxon tests are indicated in parentheses): 1.0 -1.6 $\mathrm{kHz}, 4.8 \pm 2.6 \%$ significant increase in A1 area $(p=0.002 / p=0.004) ; 1.6-2.5 \mathrm{kHz}, 6.9 \pm$ $4.8 \%$ less $\mathrm{A} 1$ area $(p=0.984 / p=0.479) ; 2.5-4.0 \mathrm{kHz}, 3.7 \pm 2.4 \%$ less $\mathrm{A} 1$ area $(p=$ $0.891 / p=0.418) ; 4.0-6.3 \mathrm{kHz}, 4.1 \pm 2.4 \%$ significant increase in $\mathrm{A} 1$ area $(p=0.022 / p=$ $0.010) ; 6.3-10 \mathrm{kHz}, 3.5 \pm 3.2 \%$ increase in A1 area $(p=0.328 / p=0.016) ; 10-15.9 \mathrm{kHz}$, $0.7 \pm 1.5 \%$ increase in A1 area $(p=0.219 / p=0.366) ; 15.9-25.2 \mathrm{kHz}, 1.2 \pm 3.1 \%$ increase in $\mathrm{A} 1$ area $(p=0.086 / p=0.116) ; 25.2-39.9 \mathrm{kHz}, 0.9 \pm 3.2 \%$ less A1 area $(p=0.445 / p=$ 0.334). C, Sound level representation in A1. Analysis of best frequency areas of A1 representation between groups ( $y$-axis) across various sound levels ( $x$-axis) revealed that frequencyspecific expansion for Signal $A$ in rats treated with RGFP966 occurred only in the representation of the best frequency determined at $70 \mathrm{~dB}$ SPL, i.e., at the unique sound level of Signal A. The expansion of Signal B occurred across all sound levels. Asterisks indicate that sound levels that were significantly increased in RGFP966-treated rats using both paired (sign test) and unpaired (Mann-Whitney-Wilcoxon) one-sided tests after Holm-Bonferroni correction ( $p$ values for the sign test/Mann -Whitney-Wilcoxon tests are indicated in parentheses): for Signal A, 70 $\mathrm{dB}, 4.1 \pm 2.4 \%$ significant increase in $\mathrm{A} 1$ area $(p=0.027 / p=0.030) ; 60 \mathrm{~dB}, 4.2 \pm 2.3 \%$ significant increase in $\mathrm{A} 1$ area $(p=0.027 / p=0.044) ; 50 \mathrm{~dB}, 0.02 \pm 2.5 \%$ nonsignificant change in $\mathrm{A} 1$ area $(p=0.055 / p=0.089) ; 40 \mathrm{~dB}, 2.9 \pm 2.2 \%$ decrease in $\mathrm{A} 1$ area $(p=$ $0.056 / p=0.084)$; for Signal B: $70 \mathrm{~dB}, 4.8 \pm 2.6 \%$ significant increase in $\mathrm{A} 1$ area $(p=$ $0.004 / p=0.004) ; 60 \mathrm{~dB}, 8.0 \pm 5.0 \%$ significant increase in $\mathrm{A} 1$ area $(p=0.041 / p=0.041)$; $50 \mathrm{~dB}, 4.7 \pm 3.6 \%$ significant increase in $\mathrm{A} 1$ area $(p=0.029 / p=0.036) ; 40 \mathrm{~dB}, 4.3 \pm 3.8 \%$ significant increase in $\mathrm{A} 1$ area $(p=0.014 / p=0.041)$. Mean differences between six pairs of RGFP966- and vehicle-treated animals (total $N=12$ ) are shown ( \pm SE). Note that the study design did not permit detection of significant differences between Signal $A$ and Signal $B$ in the identified behavioral and neural response changes between groups. Thus, the report focuses on RGFP966 versus vehicle treatment effects with respect to frequency specificity per se. 
A
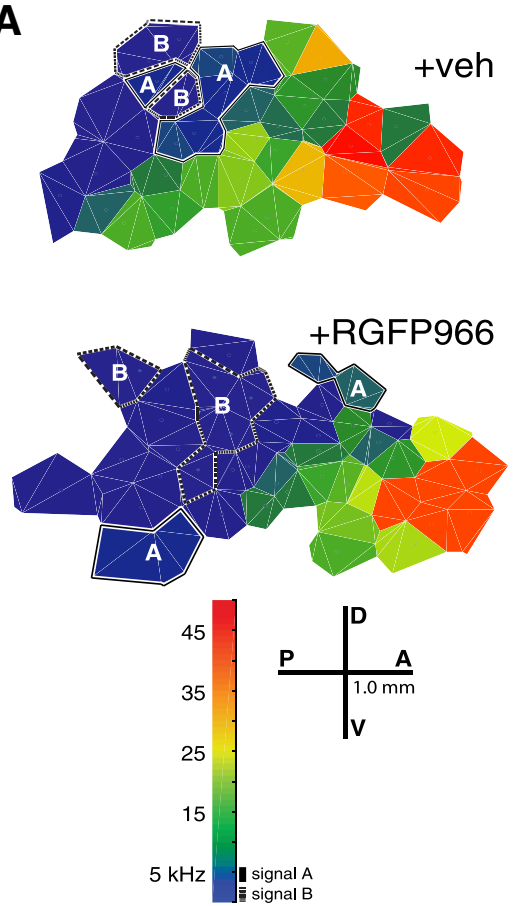

B

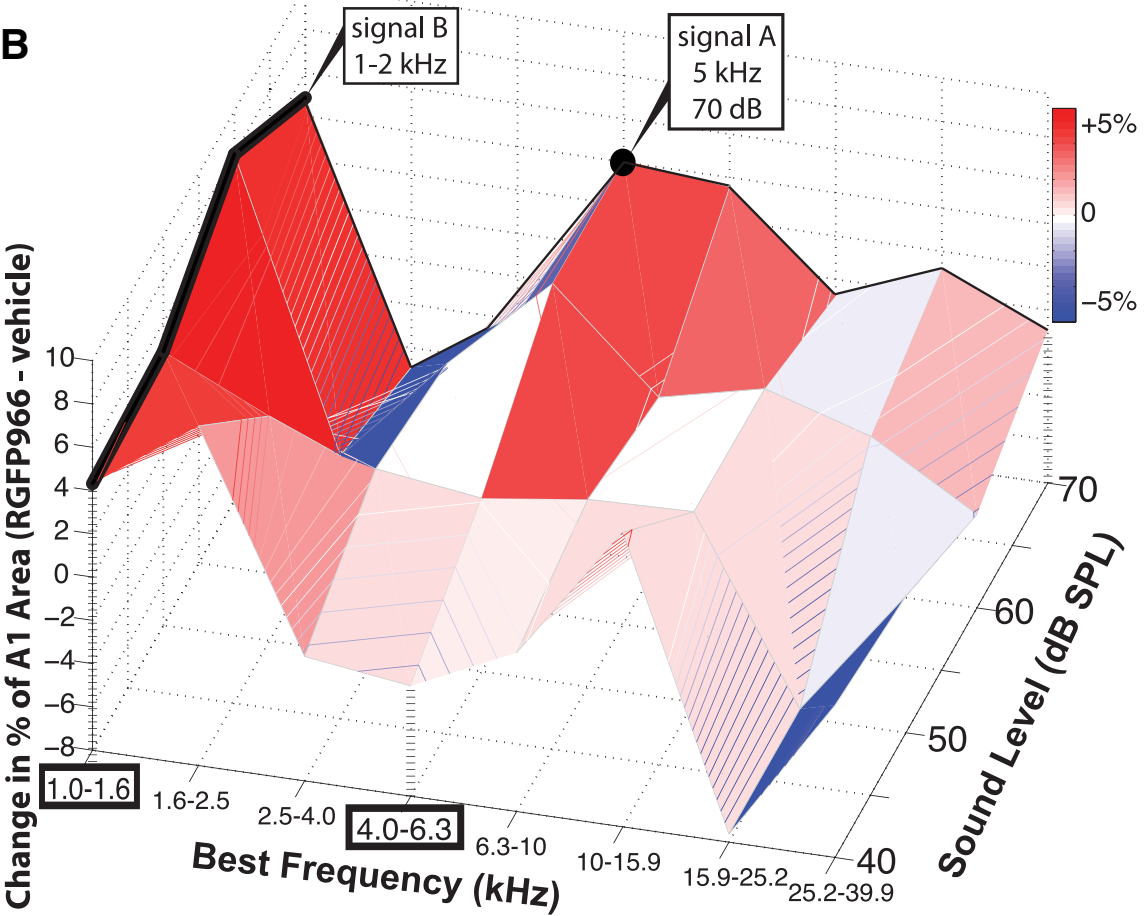

Figure 3. RGFP966 enables highly specific cortical remodeling that changes the landscape of sound representation in A1.A, Tonotopic plasticity in A1. Representative maps show sound frequency representation in A1 for a pair of performance-matched rats (+VEH, treated with vehicle treated during training; + RGFP966, treated with RGPF966 during training). Maps were constructed using Voronoi tessellation algorithms that circumscribe and denote (F tuning by colored polygons (cool colors show low CFs, whereas warmer colors show higher CFs). Note the general progression of CFs from posterior to anterior sites across the cortical surface. Solid lines outline cortical areas that represent a quarter-octave range around the Signal A frequency. Likewise, stippled lines outline cortical areas that represent a quarter-octave range around Signal B. Ruler bar shows $1.0 \mathrm{~mm}$ across the AP and DV surfaces, as indicated. B, Signal-specific map reorganization. Cortical remodeling induced by RGFP966 in A1 enables expansions (shown in red shading) in the representation of reward-predicting auditory cues, here shown as the difference in the amount of percentage increase in A1 tonotopic representation across different frequency ranges ( $x$-axis) at different sound levels ( $y$-axis). Rats treated with RGFP966 show enhanced specificity of A1 reorganization relative to vehicle-treated rats (increases greater than vehicle controls indicated by red shading and decreases less than controls by blue). Maximal expansion in A1 representation occurs for the identity of specific reward signals, e.g., sound Signal A ( $5 \mathrm{kHz}, 70 \mathrm{~dB}$, shown by the solid circle) and Signal B (1-2 kHz, indicated by the thick solid line). Note that this figure combines and expands the data shown in Figure 2.

ulation of cells. The illustration of bandwidth analysis in Figure 4 shows a symmetrical caricature of the tuning shape of group FRAs constructed from the mean BWs calculated per sounds level in each isofrequency band. Rather than the true shape of an FRA, this depiction is meant to illustrate the systematic nature of the bandwidth differences between groups, with respect to frequency and sound level.

\section{Tissue preparation}

An additional cohort of rats that were not included in the electrophysiological mapping and behavioral memory testing part of the study was also trained. On the day of the second drug treatment with vehicle $(N=6)$ or the HDAC3 inhibitor RGFP966 $(N=6)$, which was after the fifth training session, animals were deeply anesthetized by isoflurane 50 min after injection ( $4 \%$ in oxygen). They were killed by decapitation using a rat guillotine so brains could be harvested and immediately flash frozen. Horizontal sections ( $20 \mu \mathrm{m}$ thick) including the AC and dorsal hippocampus (HIPP) were collected for immunofluorescence analysis of epigenetic marks.

\section{Immunofluorescence}

Slides were fixed in $4 \%$ paraformaldehyde for $10 \mathrm{~min}$ at room temperature, blocked in $8 \%$ normal goat serum (Jackson Immunoresearch Laboratories) with $0.3 \%$ Triton X-100 in PBS, and incubated overnight at $4{ }^{\circ} \mathrm{C}$ with primary antibody in block solution, followed by a $2 \mathrm{~h}$ incubation at room temperature with secondary antibody. Primary antibodies used were acetyl-histone-H4K8 (1:1000 dilution; Cell Signaling Technology), acetyl-histone-H3K14 (1:1200; Millipore), and acetyl-histone-H2BK12 (1:1000; Abcam). Adjacent sections were labeled using primary antibody against NeuN (1:100; Millipore). The secondary antibody was goat anti-rabbit IgG-FITC
(1:1,000; Millipore Bioscience Research Reagents) or goat anti-mouse IgG-Cy3 (1:200; Millipore). All sections were counterstained with DAPI (1:25,000; Invitrogen). Immunolabeling was quantified by measuring the optical density from comparable $4 \times$ images from each animal using ImageJ software. The primary auditory cortex was defined by the area corresponding to area "Aul" as defined by Paxinos and Watson (1998) [AP, -6.5 to $-3.5 \mathrm{~mm}$; dorsoventral (DV), -3.38 to $-5.60 \mathrm{~mm}$ ] and by cell morphology and density using definitions for A1 and cortical layers as is typically described: a decreased cell density in layer $\mathrm{V}$ of primary cortex, where layer $\mathrm{V}$ is defined as $\sim 51-77 \%$ of the distance through the cortical thickness from the pial surface (Games and Winer, 1988; Weedman and Ryugo, 1996; Hefti and Smith, 2000). HIPP was defined by the area corresponding to the approximate location of CA1/CA2 (AP, -6.8 to -5.0 $\mathrm{mm}$ ) in the same dorsal-ventral sections as were collected for AC analysis for valid comparison.

\section{Determining behavioral frequency specificity}

Individual analysis. To determine the specificity of auditory memory, each animal's behavioral gradient for frequency (across the five test frequencies: 1.1, 2.4, 5.0, 10.6, and $22.4 \mathrm{kHz}$ ) was evaluated for peaks. A peak in the gradient was defined as a low point at a single test frequency that was more than a one SE decreased in latency relative to the animal's average latency across all test frequencies (i.e., the animal's baseline latency). (Note that the term "peak" is used as a convenience, as this is actually a "nadir" in latency curves.) To be defined as a peak, nearest neighboring test frequencies had to be of longer latency. This analysis allows for multiple peaks to be detected in 


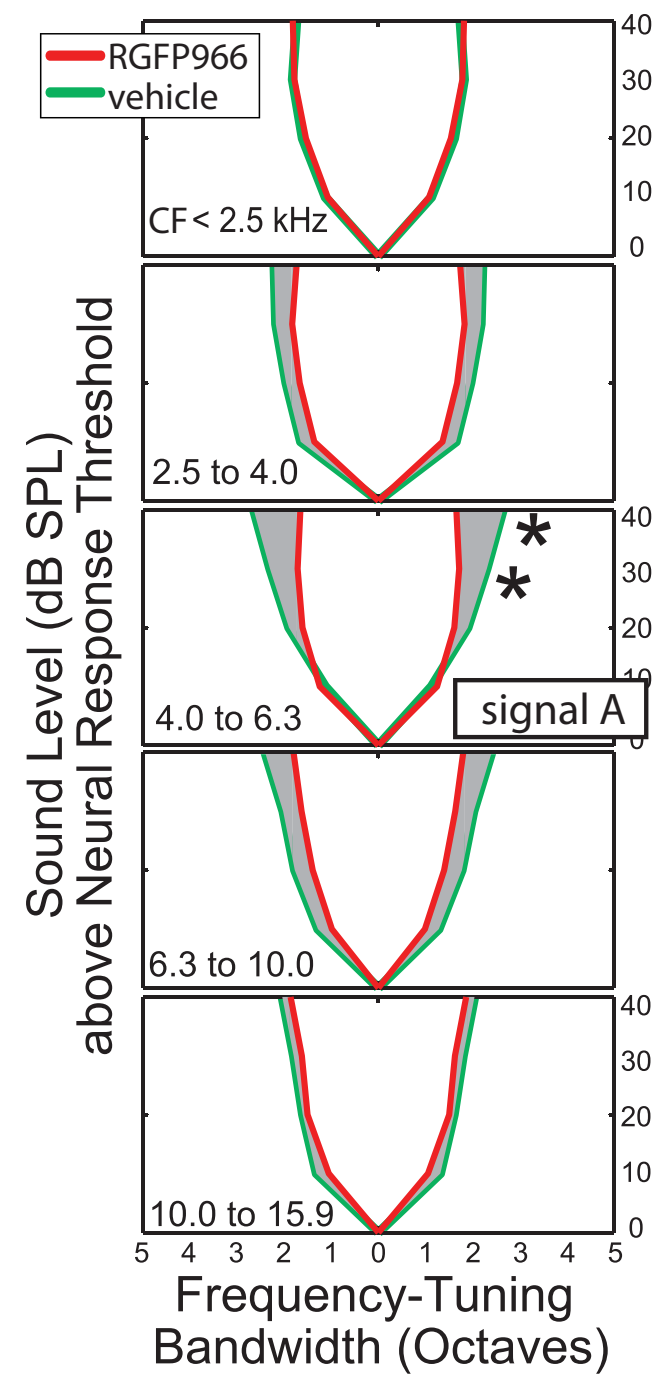

Figure 4. RGFP966 enables highly specific cortical remodeling: reduced tuning bandwidth. A second form of $A 1$ representational plasticity reduces tuning bandwidth only in neurons tuned near an auditory signal associated with reward. Panels show tuning curve means of populations of neurons with CFs tuned within the range indicated in the lower left. All bandwidths are shown in decibel level relative to $\mathrm{CF}$ threshold. Asterisks in the middle panel indicate significant decreases in bandwidth (Mann-Whitney test, $p<0.05$ ), which occurred for sites tuned near Signal $A$ at $30 \mathrm{~dB}$ ( $\sim 0.5$ octave decrease in tuning bandwidth) and $40 \mathrm{~dB}$ ( $\sim 1.0$ octave decrease in tuning bandwidth) above threshold. Detection of changes in bandwidth for Signal $B$ were not possible in this dataset due to a sampling floor effect on the low-frequency side of the Signal B frequency range.

individual behavioral gradients, which made it possible, e.g., to detect memory for either Signal A or Signal B, or for both signals.

Group analysis. To determine the effects of HDAC3i on specific memory encoding, the individual normalized behavioral gradient for an animal treated with vehicle was subtracted from the normalized behavioral gradient of a performance-matched HDAC3i-treated animal (i.e., pairs of HDAC3i minus vehicle animals). There were six pairs of performancematched animals (total, $N=12$ ), whose difference gradients were averaged to find peaks in the mean difference gradient. A peak in the difference gradient was defined as described above for individual animal analyses to indicate which frequencies HDAC3i-treated animals had better remembered relative to vehicle-treated animals. Figure 2 (inset) also shows normalized behavioral gradients that were averaged within a group, without respect to performance-matched pairs (HDAC3i group, $N=6$; vehicle group, $N=$ 6), to show actual bar-press latencies for each group.

Statistics. A paired-sample $t$ test or $Z$ test and nonparametric sign test (for performance-matched paired data) or Mann-Whitney-Wilcoxon (for unpaired data) tests were used to determine significant differences in group data with $\alpha$ levels held at 0.05 . Nonparametric statistics were used for small-sample behavioral analysis, as described in Results. Pearson's correlation coefficient was used for brain versus behavior analyses. The Holm-Bonferroni method appropriately adjusted for multiple comparisons across sound frequencies. Group sizes were chosen to establish power at $0.80(\alpha=0.05)$.

\section{Results \\ HDAC inhibitor RGFP966 alters the behavioral specificity of memory formation}

To test the hypothesis that HDAC inhibition has a key function in specific information encoding, we used the class I HDAC inhibitor called RGPF966. In a previous study, we established that RGFP966 crosses the blood-brain barrier with a brain/plasma ratio of 0.45 (Malvaez et al., 2013). In a substrate-dependent biochemical assay using purified recombinant HDACs, RGFP966 was found to be specific for HDAC3, with an $\mathrm{IC}_{50}$ value of 0.08 $\mu \mathrm{M}$ and no effective inhibition of any other HDAC at concentrations of up to $15 \mu \mathrm{M}$ (Malvaez et al., 2013). Malvaez et al., (2013) found that a dose of $10 \mathrm{mg} / \mathrm{kg}$ resulted in concentrations above the $\mathrm{IC}_{50}$ value between $30 \mathrm{~min}$ and $1 \mathrm{~h}$ after delivery (yet 10 -fold lower than the $15 \mu \mathrm{M}$ concentration at which HDAC1 and HDAC2 may become engaged), indicating this dose is ideal for studying posttraining effects of RGFP966 on memory formation. Here, we confirmed that RGFP966 at a $10 \mathrm{mg} / \mathrm{kg}$ dose penetrates the blood-brain barrier into rat auditory cortex with typical pharmacokinetics, which together establish feasibility for the modulation of A1 plasticity due to action in the auditory cortex (Fig. 1A).

RGFP966 or vehicle was injected into rats learning to associate specific sound frequencies with reward in an auditory instrumental conditioning paradigm. There were two auditory signals associated with reward (Fig. 1B). The first sound, Signal A (a $5.0 \mathrm{kHz}$ pure tone presented at $70 \mathrm{~dB}$ ), signaled reward delivery contingent on a bar-press response. The second sound, Signal B (generated by a mechanical lever that delivered the reward through a port), signaled immediate availability of reward. Prior studies using this training protocol found that learning can produce strong memories and $\mathrm{A} 1$ plasticity for the $5.0 \mathrm{kHz}$ pure tone, i.e., Signal A (Bieszczad and Weinberger, 2010a,b). Furthermore, HDAC inhibition by RGFP966 may promote acetylation during consolidation of auditory memory. Lysine 8 on histone 4 (H4K8) and lysine 14 on histone 3 (H3K14) are known targets of HDAC3, whereas lysine 12 on histone $2 \mathrm{~B}$ (H2BK12) is not a known target. Immunofluorescence analyses did not reveal statistically significant increases in acetylation at two potential acetylation sites $(1.2 \pm 1.6 \%$ relative decrease of $\mathrm{H} 3 \mathrm{~K} 14$-Ac in primary auditory cortex, $t$ test, $p=0.78 ; 3.1 \pm 3.5 \%$ relative increase in CA1 of the hippocampus, $t$ test, $p=0.19 ; 8.0 \pm 6.6 \%$ relative decrease of $\mathrm{H} 2 \mathrm{BK} 12-\mathrm{Ac}$ in A1, $t$ test, $p=0.89 ; 2.7 \pm 7.5 \%$ relative decrease in HIPP, $t$ test, $p=0.64 ; N=6$ rats per group; $N=3$ replicates per rat). However, there was evidence of tendency for increased acetylation at H4K8 in A1 (but not HIPP) in RGFP966-treated animals relative to vehicle-treated controls $(4.9 \pm 3.6 \%$ relative increase of $\mathrm{H} 4 \mathrm{~K} 8$-Ac in A $1, t$ test, $p=0.09 ; 0.4 \pm 4.3 \%$ increase in HIPP, $t$ test, $p=0.46$ ), suggesting that the locus of effect might be confined to a more specific cortical region within A1.

After 2 weeks of daily training and RGFP966 administration, we found that HDAC inhibition by RGFP966 did not affect acquisition or final performance level relative to vehicle-treated controls (Fig. 1C). However, a behavioral test for the sound specificity of the auditory memory formed revealed that the group 
treated with RGFP966 remembered the signal sounds with higher specificity for acoustic frequency. Relative to a performancematched vehicle-treated group, the behavioral gradient for frequency specificity at memory test showed that RGFP966 induced more specific memory for Signal A (Fig. 2A). In addition, we discovered that RGFP966 also permitted the incorporation of additional information for a second frequency, here called Signal B. In contrast, no vehicle-treated animals developed memory for both frequency Signals A and B. Therefore, RGFP966 enabled the formation of a more specific auditory memory as well as memory that incorporated information for not just one, but two sound frequencies that were associated with reward.

\section{RGFP966 alters signal-specific primary auditory cortical plasticity}

Evidence for the neural mechanism of increased behavioral specificity was found in A1. We discovered that RGFP966 enhanced the cortical representations of the two reward-related signal sounds. Indeed, the tonotopic areas of both the Signal A and Signal B frequencies increased in RGFP966-treated animals relative to vehicle-treated controls (Fig. 2B). Furthermore, in addition to a specific effect on sound frequency map expansion in A1, the RGFP966 effect extended to an additional acoustic parameter: sound loudness. This was detectable because Signal A was unique in its sound level during training, i.e., it was always presented at $70 \mathrm{~dB}$. A1 neurophysiology was determined at multiple sound levels, including $70 \mathrm{~dB}$, thereby enabling analysis of neural specificity for sound loudness. Remarkably, RGFP966 enhanced the expansion for the $5.0 \mathrm{kHz}$ (Signal A) frequency only in its representation in A1 near the $70 \mathrm{~dB}$ SPL (Fig. 2C). The high signal specificity for both the frequency and loudness of Signal A was in contrast to Signal B, which was found to have a specific effect on A1 sound frequency representation, but a general effect on the representation of sound level. The specific frequency representation of Signal B (near $1.1 \mathrm{kHz}$ ) was significantly greater across all sound levels in A1, i.e., the effect generalized across sound loudness (Fig. 2C). A potential explanation for the lack of level-specific effects for Signal B is that it was not learned at a unique sound level during training. Indeed, the general effect on A1 level representation for Signal B need not be a failure of plasticity, but rather an induction of plasticity that accurately reflects the characteristic sensory features of Signal B (e.g., as a single specific frequency across a broad sound level). Together, these neurophysiological findings suggest that the effect of RGFP966 on A1 was to enable plasticity that accurately and specifically captured many acoustic characteristics of the cues associated with reward.

We next determined whether behavioral frequency specificity was linked to the magnitude of frequency-specific A1 area gain. Indeed, there was a significant positive brain-behavior correlation: the greater the Al expansion, the more specific the auditory memory. A positive curvilinear relationship indicated that the stronger the specificity of signal frequency memory (revealed as the peak in behavioral response to Signal B at $1.1 \mathrm{kHz}$ ), the greater the specific frequency representation in A1 (Pearson's correlation coefficient, $\left.R^{2}=0.6775 ; p<0.001\right)$. Interestingly, this relationship was strongest at the level of CF, which describes A1 frequency representation at evoked threshold, rather than representation at $\mathrm{BF}\left(R^{2}=0.3196 ; p=0.055\right)$, which predicted the observed nonsignificant correlation for the other, Signal A frequency $\left(R^{2}=0.2647 ; p=0.44\right)$, for which A1 had reorganized only at BF (i.e., near $70 \mathrm{~dB}$ ). These data support the hypothesis that RGFP966 mediates a transformation of learning experiences into highly specific long-term memories via modulation of the sensory cortical plasticity of sound representation in A1.

Collectively, it appears that RGFP966 enables A1 remodeling in a multidimensional way to increase cortical representations of multiple sounds and sound features that are associated with reward (Fig. 3). To determine the extent of this apparent effect of RGFP966 to regulate A1 plasticity, we studied another form of representational plasticity in $\mathrm{Al}$ : breadth of frequency-tuning bandwidth. The different forms of representational plasticity (map vs BW changes) are important to distinguish since they may have distinct functional importance for the detection and discrimination of behaviorally relevant cues, respectively (Scheich et al., 2011). Thus, differences in receptive field bandwidth between RGFP966- and vehicle-treated animals were also examined. Here, we found that A1 sites with CF tuning to within half an octave around Signal A $(5.0 \mathrm{kHz})$ had decreased breadth of tuning bandwidth with RGFP966 treatment (relative to vehicle controls). Signal B fell on the low-frequency edge of detection for calculating BW, so we were unable to determine whether similar decreases in bandwidth were present for the Signal B sound frequency $(1.1 \mathrm{kHz})$. Nevertheless, the effect of RGFP966 on representation of Signal A was to sharpen A1 frequency tuning by as much as an octave (Fig. 4). We conclude from these data that RGFP966 affected cortical remodeling of (at least) two forms: signal-specific representational expansion and decreased frequency-tuning bandwidth.

\section{Discussion \\ HDAC inhibition enhances memory and cortical plasticity with added auditory detail}

Animals treated with RGFP966 after learning an association between sound and reward remembered more signals related to reward and remembered highly specific auditory features of those signals. Evidence for the role of RGFP966 to enhance the encoding of sensory information emerged in two ways. First, RGFP966 enabled memory that was stronger, as revealed by shortened latency behavioral responses to signal sounds. Second, RGFP966 induced memory that was more specific. Behavioral responses indicated memory for not just one signal sound (as in the vehicletreated condition), but two signal sounds associated with reward. Furthermore, this effect of RGFP966 was not evident in acquisition or absolute levels of achieved performance in the task. Rather the effect of RGFP966 was on the specificity of auditory information encoded, which was revealed in a subsequent memory test for the frequency specificity of long-term memory. Thus, RGFP966 appears to alter information encoding (what and how much is encoded as a signal) without an effect on associative mechanisms (those which link the signal with reward) per se.

At the neural level, the effect of RGFP966 to alter frequency specificity was recapitulated in the apparent frequency-specific tonotopic plasticity for the remembered sound signals. Signals A and B both had enlarged cortical representations. Tonotopic plasticity was highly specific for the signals' sound frequencies, and it was also highly specific for the signals' sound levels. Future experiments are necessary to determine whether the specificity of A1 plasticity for sound level is behaviorally relevant. Learninginduced A1 plasticity can be highly specific for a variety of features of sound, including sound level (Polley et al., 2006), timing (Bao et al., 2004), and even location (Lee and Middlebrooks, 2011), among others (Scheich et al., 2011). Overall, the results suggest that HDAC inhibition opens the gates to neural processes enabling information encoding of highly specific sensory features of signals associated with reward. A general prediction ex- 
tending from these findings is that HDACs might regulate what and how much information of any type will become encoded into memory.

\section{Informational capture by HDAC inhibition}

The current report of behavioral and neural effects on auditory memory formation and A1 plasticity suggest that HDACs can regulate information encoding. Indeed, sensory cortical plasticity and its epigenetic regulation suggest key roles for both mechanisms in the systems-level formation and consolidation of information-rich memory. An HDAC-mediated role in the specificity of auditory memory and A1 plasticity could exemplify a fundamental principle that explains reported effects of HDAC3 in hippocampus-dependent memory. Synthesis of prior findings with this report introduces a new hypothesis: HDACs regulate informational capture. Here we define informational capture as the transformation of a sensory "snapshot" from the immediate short-term memory of a current experience into a persistent long-term memory that retains the perceptual vividness of the original experience.

A general function of HDACs might be to regulate the scope of capture from perception and short-term memory into long-term memory. Thus, HDAC inhibitors would contribute to concurrently enhancing and inducing memory for multiple and highly specific sensory features of behaviorally relevant signals. Furthermore, we assign the predicted influence of HDACs to associative processes per se, as opposed to attention-related or perceptual processes active during the task (i.e., because RGFP966 is administered after training and therefore not present in the system during learning sessions). The effect of HDAC inhibition to enhance memory specificity could explain other reports showing longterm memory enabled by RGFP966 during subthreshold learning for object location (Malvaez et al., 2013, their supplemental Fig. 2). Having memory for these precise stimuli that are rich in detail and multisensory information would enable their correct recognition and discrimination at testing. Our interpretation of such data is that RGFP966 facilitates task performance by enabling memory to form and consolidate with exceptional sensory detail.

\section{Epigenetic mechanisms regulate information encoding}

Epigenetic regulation of neural plasticity in adult A1 opens questions of how chromatin modification mechanisms such as histone acetylation might act in the cortex as a whole to altogether contribute to information processing and depth of encoding in memory formation. Future experiments could reveal that memory for a specific frequency facilitates learning of a two-tone auditory discrimination in which one tone signals reward ("tone+" cue) and the other the unavailability of reward (a "tone-" cue). In this case, acquisition and achieved performance levels might also be facilitated with RGFP966 because specific information about frequency is necessary to perform correct frequency discriminations.

Although identifying molecular mechanisms was beyond the scope of the present study, that epigenetic regulation per se is key for controlling the specificity of learned information is supported by complementary evidence that DNA methylation can alter auditory discrimination behavior in rats (Day et al., 2013) and olfactory discrimination behavior in honeybees (Biergans et al., 2012). Subsequent analyses could determine the key genes regulated by a family of epigenetic players, including HDAC3 (considering that RGFP966 may preferentially affect HDAC3 activity), that ultimately affect the specificity of memory. The auditory model used here is unique in that it can be used to identify an epigenetic mechanism for memory formation at the level of cortical plasticity, which offers an approach in future studies to detect critical changes at an underlying level in gene expression profiles by targeting those neurons in A1 identified by shifts in receptive field properties within regions of cortical overrepresentation for reward-related sounds. Tools are becoming available to target the critical cell types, cortical columns, and layers of A1 tissue that have undergone plasticity, so future studies can begin to establish the molecular and genetic mechanisms for learning-induced sensory cortical plasticity that appears to be regulated by HDACs in the formation of sensory-specific memory. Such studies will be necessary to prove that HDAC 3 is indeed key to information processing in the auditory cortex, as suggested by results generated from the use of the HDAC3 selective inhibitor RGFP966 in this study.

\section{Conclusions}

Overall, the findings show that an inhibitor selective for HDAC3 can be used to enable representational plasticity in sensory cortex that underlies memory for highly specific auditory information. As such, HDAC3 inhibitors could be key target drugs for altering the threshold of memory induction in the transformation from immediate perception to enduring memory. We introduce the hypothesis of "informational capture," whereby currently available perceptual information is transformed by HDACdependent mechanisms from short- to long-term memory. It is possible that HDAC3 (or other HDACs of the class I type) regulates the amount of information entered into memory during initial memory encoding, memory consolidation, or both. The proposed function of these HDACs in sensory system plasticity and consolidation is particularly relevant to clinical conditions that suffer from failures in the specificity of encoded information. For example, combined behavioral and pharmacological approaches are provocative for their prospective ability to rescue or enable auditory learning, comprehension, or language acquisition, which demand auditory signal specificity to successfully attribute significance to particular sounds and their meaning. Benefit could be gained from behavioral therapies with concurrent use of class I HDAC-targeting drugs. We propose here that HDACs, and perhaps HDAC3 specifically, will gate memory for informative cues and their complex sensory features in any associative learning experience.

\section{References}

Bakin JS, Weinberger NM (1990) Classical conditioning induces CSspecific receptive field plasticity in the auditory cortex of the guinea pig. Brain Res 536:271-286. CrossRef Medline

Bao S, Chang E, Woods J, Merzenich MM (2004) Temporal plasticity in the primary auditory cortex induced by operant perceptual learning. Nat Neurosci 7:974-1055. CrossRef

Biergans SD, Jones JC, Treiber N, Galizia CG, Szyszka P (2012) DNA methylation mediates the discriminatory power of associative long-term memory in honeybees. PLoS One 7:e39349. CrossRef

Bieszczad KM, Weinberger NM (2010a) Representational gain in cortical area underlies increase of memory strength. Proc Natl Acad Sci U S A 107:3793-3798. CrossRef

Bieszczad KM, Weinberger NM (2010b) Remodeling the cortex in memory: increased use of a learning strategy increases the representational area of relevant acoustic cues. Neurobiol Learn Mem 94:127-171. CrossRef

Bieszczad KM, Weinberger NM (2012) Extinction reveals that primary sensory cortex predicts reinforcement outcome. Eur J Neurosci 35:598-613. CrossRef

Bieszczad KM, Miasnikov AA, Weinberger NM (2013) Remodeling sensory cortical maps implants specific behavioral memory. Neurosci 246:40-51. CrossRef

Day JJ, Childs D, Guzman-Karlsson MC, Kibe M, Moulden J, Song E, Tahir A, 
Sweatt JD (2013) DNA methylation regulates associative reward learning. Nat Neurosci 16:1445-1452. CrossRef Medline

Froemke RC, Carcea I, Barker AJ, Yuan K, Seybold BA, Martins ARO, Zaika $\mathrm{N}$, Bernstaein H, Wachs M, Levis PA, Polley DB, Merzenich MM, Schreiner CE (2013) Long-term modification of cortical synapses improves sensory perception. Nat Neurosci 16:79-88. Medline

Games KD, Winer JA (1988) Layer V in rat auditory cortex: Projections to the inferior colliculus and contralateral cortex. Hear Res 34:1-25. CrossRef Medline

Gonzalez-Lima F, Scheich H (1986) Neural substrates for tone-conditioned bradycardia demonstrated with 2-deoxyglucose. II. Auditory cortex plasticity. Behav Brain Res 20:281-374. CrossRef

Hefti BJ, Smith PH (2000) Anatomy, physiology, and synaptic responses of rat layer $\mathrm{V}$ auditory cortical cells and effects of intracellular GABAA blockade. J Neurophysiol 83:2626-2638. Medline

Lee CC, Middlebrooks J (2011) Auditory cortex spatial sensitivity sharpens during task performance. Nat Neurosci 14:108-122. CrossRef

Malvaez MM, McQuown SC, Rogge GA, Astarabadi M, Jacques V, Carreiro S, Rusche JR, Wood MA (2013) From the Cover: HDAC3-selective inhibitor enhances extinction of cocaine-seeking behavior in a persistent manner. Proc Natl Acad Sci U S A 110:2647-2652. CrossRef

McQuown SC, Wood MA (2011) HDAC3 and the molecular brake pad hypothesis. Neurobiol Learn Mem 96:27-34. CrossRef

McQuown SC, Barrett RM, Matheos DP, Post RJ, Rogge GA, Alenghat T, Wood MA (2011) HDAC3 is a critical negative regulator of long-term memory formation. J Neurosci 31:764-774. CrossRef

Merzenich MM, Knight PL, Roth GL (1975) Representation of the cochlea within the primary auditory cortex in the cat. J Neurophysiol 38:231-249. Medline

Paxinos G, Watson C (1998) The rat brain. San Diego: Academic.

Peixoto L, Abel T (2013) The role of histone acetylation in memory formation and cognitive impairments. Neuropsychopharmacology 38:62-76. CrossRef

Pienkowski M, Eggermont JJ (2011) Cortical tonotopic map plasticity and behavior. Neurosci Biobehav Rev 35:2117-2128. CrossRef

Polley DB, Steinberg E, Merzenich MM (2006) Perceptual learning directs auditory cortical map reorganization through top-down influences. J Neurosci 26:4970-5052. CrossRef
Polley DB, Read HL, Storace DA, Merzenich MM (2007) Multiparametric auditory receptive field organization across five cortical fields in the albino rat. J Neurophysiol 97:3621-3638. CrossRef

Rumbaugh G, Sillivan SE, Ozkan ED, Rojas CS, Hubbs CR, Aceti M, Kilgore M, Kudugunti S, Puthanveettil SV, Sweatt JD, RuscheJ, Miller CA (2015) Pharmacological selectivity within class I histone deacetylases predicts effects on synaptic function and memory rescue. Neuropsychopharmacology 40:2307-2316. CrossRef Medline

Rutkowski RG, Weinberger NM (2005) Encoding of learned importance of sound by magnitude of representational area in primary auditory cortex. Proc Natl Acad Sci U S A 102:13664-13669. CrossRef

Rutkowski RG, Miasnikov AA, Weinberger NM (2003) Characterisation of multiple physiological fields within the anatomical core of rat auditory cortex. Hear Res 181:116-130. Medline

Scheich H, Ohl FW (2010) A semantic concept of auditory cortex function and learning. In: The auditory cortex, pp 369-387. Boston: Springer.

Scheich H, Brechmann A, Brosch M, Budinger E, Ohl FW, Selezneva E, Stark H, Tischmeyer W, Wetzel W (2011) Behavioral semantics of learning and crossmodal processing in auditory cortex: the semantic processor concept. Hear Res 271:3-18. Medline

Schreiner CE, Polley DB (2014) Auditory map plasticity: diversity in causes and consequences. Curr Opin Neurobiol 24:143-156. CrossRef Medline

Schulte M, Knief A, Seither-Preisler A, Pantev C (2002) Different modes of pitch perception and learning-induced neuronal plasticity of the human auditory cortex. Neural Plast 9:161-175. CrossRef

Stefanko DP, Barrett RM, Ly AR, Reolon GK, Wood MA, McGaugh JL (2009) Modulation of long-term memory for object recognition via HDAC inhibition. Proc Natl Acad Sci U S A 106:9447-9452. CrossRef

Weedman DL, Ryugo DK (1996) Projections from auditory cortex to the cochlear nucleus in rats: synapses on granule cell dendrites. J Comp Neurol 371:311-324. Medline

Weinberger NM (2007) Associative representational plasticity in the auditory cortex: a synthesis of two disciplines. Learn Mem 14:1-16. Medline

Weinberger NM, Miasnikov AA, Bieszczad KM, Chen JC (2013) Gamma band plasticity in sensory cortex is a signature of the strongest memory rather than memory of the training stimulus. Neurobiol Learn Mem 104: 49-63. CrossRef 